autophagy with Chloroquine (CQ). BKM120/CQ combination resulted in a cell viability increase suggesting that autophagy activation is detrimental to ependymoma survival. Gene expression analysis is ongoing to further elucidate downstream pathway targets in ependymoma cells. CONCLUSION: PI3K pathway inhibition triggers cellular mechanisms that oppose survival responses in ependymoma cells. The consequent adverse effects in these tumours implicates the pathway's oncogenic role, highlighting its therapeutic value for the clinical management of ependymoma.

\section{EPND-05. LYSINE SPECIFIC DEMETHYLASE-1 (LSD-1) INHIBITOR SYC-836 IN COMBINATION WITH RADIATION PROLONGS ANIMAL SURVIVAL IN PATIENT-DERIVED ORTHOTOPIC XENOGRAFT (PDOX) MODEL OF POSTERIOR FOSSA EPENDYMOMA \\ Sibo Zhao, Huiyuan Zhang, Lin Qi, Holly Lindsay, Yuchen Du,} Mari Kogiso, Frank Braun, Sarah Injac, Lazlo Perlaky, Donald W. Parsons, Murali Chintagumpala, Adekunle Adesina, Yongcheng Song, and XiaoNan Li; Baylor College of Medicine, Houston, TX, USA.

BACKGROUND: Recent molecular subgrouping of ependymoma (EPN) has identified one group, posterior fossa A (PFA), to have one of the worst prognosis and driven by epigenetic changes, suggesting targeting epigenetic changes in PFA EPN can potentially be effective. In this study, we examined the therapeutic efficacy of SYC-836, a novel LSD-1 inhibitor compound developed at Baylor College of Medicine, in PDOX models of posterior fossa EPN. METHODS: To examine in vitro anti-tumor activities, paired primary cultured cells (both as attached cells and neurospheres) from a posterior fossa EPN cell line (ICb-4423EPN) were subjected to SYC-836 at various concentrations $(0-25 \mathrm{uM})$. Cell viability and proliferation were measured over 14 days. To validate the drug's in vivo efficacy, two established posterio fossa EPN PDOX models, ICb-4423EPN and ICb-2002EPN, were utilized. 40 eight weeks old SCID mice per model were implanted with tumor cells and divided into 4 treatment groups each: 1) control, 2) radiation, 3) SYC836 only, and 4) combination (radiation + SYC-836). Animal survival times were analyzed using log rank analysis. Changes of histone lysine methylation were examined through western hybridization. RESULTS: SYC-836 demonstrated effective cell killing in vitro in both time- and dose-dependent manner. In vivo experiment was completed in 1 of the 2 EPN PDOX models with the second model ongoing. There were no survival benefit with either XRT only $(P=0.205)$ or SYC-836 only $(P=0.186)$ when compared to the control group; however, when used in combination, the treatment strategy lead to significant improvement in animal survival $(P=0.004)$. SYC-836 was well tolerated in mice. CONCLUSION: Our data showed that combining SYC-836 with current standard therapy of radiation synergistically prolongs animal survival significantly. SYC-836 may have a role in the clinical setting by either reducing radiation dosages, or be an adjuvant agent to other chemotherapy drugs in our treatment approach for ependymoma.

\section{EPND-06. IDENTIFICATION OF DISTINCT MOLECULAR} CHARACTERISTICS IN PEDIATRIC SPINAL EPENDYMOMA

Anbarasu Lourdusamy ${ }^{1}, \mathrm{Li} \mathrm{Luo}^{2}$, Lisa Storer ${ }^{1}$, Kenneth Cohen ${ }^{2}$, Linda Resar ${ }^{2}$, and Richard Grundy ${ }^{1}$; ${ }^{1}$ Children's Brain Tumour Research Centre, School of Medicine, University of Nottingham, Nottingham, UK, ${ }^{2}$ The Johns Hopkins University School of Medicine, Baltimore, MD, ISA.

Pediatric spinal ependymomas (pSP) are exceedingly rare central nervous system (CNS) tumors. Children with these tumours are likely to experience a more aggressive disease course, with a higher rate of local failure following treatment, and a higher rate of CNS metastasis, compared to adults. Because these tumours are uncommon, the molecular basis of pSP is poorly characterized. Microarray analysis of 66 pediatric ependymomas showed distinct expression patterns and detected up-regulation of several genes involved in development-related processes for spinal tumours. In addition, multiple biological processes that are less known for their involvement in pSP, oxidative phosphorylation, cellular respiration, electron transport, and cofactor metabolic process were also detected. When we compared the expression profiles of pSP to adult spinal ependymomas (aSP), we found a very low number of differentially expressed genes $(12.5 \%)$ shared between pSP and aSP. Analysis of microRNA (miRNA) expression arrays on an overlapping cohort of 49 patients showed that six miRNAs (miR-196a, miR-27b, miR$23 \mathrm{~b}$, miR-144*, and miR-10a and 10b) were up-regulated and four (miR$26 \mathrm{a}$, and miR-885-5p, miR-124, and miR-153) were down-regulated in pSP The integrative miRNA-mRNA network and predicted miRNA target analysis revealed that the miR-23-27 cluster (miR23b/miR27b) coordinately repressed expression of cell differentiation genes (ACVR1C, CBFA2T3 GPSM1, and PKDCC), and the miR-10 family (miR10a/10b) that reside within the Hox clusters of developmental regulators, repressed expression of CAM2, HDAC4, IL1RAPL1, and MTSS1L. Furthermore, these miRNAmRNA interactions were not detected in other cancers when we conducted the TCGA data analysis across ten different cancer types. Further investigation of gene expression and DNA methylation profiles in a larger cohort of pediatric ependymomas is on going, and will establish specific molecular characteristics of pSP. The identification of these candidate genes/miRNAs provides an evidence-based rationale for functional studies that will help to interrogate the initiation and progression of pSP.

\section{EPND-07. MOLECULAR HETEROGENEITY AMONG PEDIATRIC POSTERIOR FOSSA EPENDYMOMA}

Kristian Pajtler ${ }^{1}$, Ji Wen ${ }^{2}$, Martin Sill ${ }^{1}$, Tong Linn ${ }^{2}$, Jens Hübner ${ }^{1}$, Vijay Ramaswamy ${ }^{3}$, Chandanamali Punchihewa ${ }^{2}$, David Jones ${ }^{1}$, Hendrik Witt ${ }^{1}$, Lukas Chavez ${ }^{1}$, Ruth Tatevossian ${ }^{2}$, Richard Grundy ${ }^{4}$, Thomas Merchant ${ }^{2}$, Michael Taylor ${ }^{3}$, Stefan Pfister ${ }^{1}$, Andrey Korshunov ${ }^{1}$, Marcel Kool ${ }^{1}$, and David Ellison ${ }^{2} ;{ }^{1}$ German Cancer Research Center, Heidelberg, Germany, ${ }^{2}$ St. Jude Children's Research Hospital, Memphis, TN, USA, ${ }^{3}$ Hospital for Sick Children, Toronto, ON, Canada, ${ }^{4}$ Queen's Medical Center, Nottingham, UK.

Previously, we have identified nine distinct molecular groups of ependymoma across all age groups, three in each major anatomical compartment of the CNS: spinal, posterior fossa, and supratentorial. These nine molecular groups are genetically, epigenetically, transcriptionally, demographically, and clinically distinct. Pediatric intracranial ependymomas are either affiliated with the RELA and YAP1 supratentorial groups or with the posterior fossa PFA group. RELA and YAP1 ependymomas harbor recurrent and distinct fusion genes that drive the disease, but PFA ependymomas lack significant recurrent mutations, and the specific oncogenic events underlying these tumors remain undefined. Observing distinct outcomes among children with PFA ependymomas, we tested the hypothesis that further molecular diversity with clinical utility, including possible novel genetic alterations, may exist among these tumors. Genome-wide DNA methylation profiles of 681 pediatric PFA ependymomas were analyzed by unsupervised consensus hierarchical clustering and t-distributed stochastic neighbor embedding (tsne), which identified not only three major distinct molecular subgroups of PFA ependymoma: PFA-1, PFA-2, and PFA-3, but additional small subgroups that segregate within PFA-1 and PFA-2. Subgroups were characterized by distinct clinical and genetic characteristics; most PFA-3 tumors harbored 1q gain and occurred in children significantly older than those with PFA-1 and PFA-2 tumors. Some of the small subgroups demonstrated distinctive copy number alterations or gene mutations, e.g. H3 K27M. Outcome data revealed considerable heterogeneity among the different subgroups, overall survival at 5 years was $>90 \%$ for one subgroup, but $<20 \%$ for two others. We conclude that further molecular refinement of pediatric PFA ependymomas has clinical utility and the potential for enhanced risk assessment or therapeutic stratification. In addition, identification of PFA subgroups and further molecular heterogeneity within these subgroups may help to identify the drivers of PFA ependymomas.

\section{EPND-08. EPIGENETIC H3K27ME3 DEREGULATION IN PEDIATRIC} POSTERIOR FOSSA EPENDYMOMAS

Sriram Venneti ${ }^{1}$, Jill Bayliss ${ }^{1}$, Piyali Mukherjee ${ }^{2}$, Chao Lu' ${ }^{9}$, Siddhant Jain ${ }^{3}$, Chan Chung $^{1}$, Pooja Panwalkar ${ }^{1}$, Daniel Martinez $z^{4,7}$, Benjamin Sabari ${ }^{9}$, Ashley Margol ${ }^{5}$, Melike Pekmezci ${ }^{6}$, Marcin Cieslik ${ }^{1}$, Benita Tamrazi ${ }^{5}$, Benjamin Garcia ${ }^{7}$, Mariarita Santi ${ }^{4,7}$, Peter Lewis ${ }^{3}$, Cynthia Hawkins ${ }^{8}$, David Allis ${ }^{9}$, Arul Chinnaiyan ${ }^{1}$, and Alexander Judkins ${ }^{5} ;{ }^{1}$ University of Michigan, Ann Arbor, MI, USA, ${ }^{2}$ Weill Medical College of Cornell University, New York, NY, USA, ${ }^{3}$ University of Wisconsin, Madison, WI, USA, ${ }^{4}$ Children's Hospital of Philadelphia, Philadelphia, PA, USA, ${ }^{5}$ Children's Hospital Los Angeles, Los Angeles, CA, USA, ${ }^{6}$ University of California, San Francisco, San Francisco, CA, USA, ${ }^{7}$ University of Pennsylvania, Philadelphia, PA, USA, ${ }^{8}$ The Hospital for Sick Children, Tornoto, ON, Canada, ${ }^{9}$ The Rockefeller University, New York, NY, USA.

Ependymomas in children occur most commonly in the posterior fossa of the brain and cause significant morbidity and mortality. The pathogenesis of these tumors remains obscure as recent whole genome and whole exome sequencing efforts have not yielded recurrent genetic mutations. These tumors exhibit abnormal $\mathrm{CpG}$ island methylation, suggesting that epigenetic alterations may be a significant driver. To gain insights into the epigenetics of childhood ependymomas, we performed mass spectroscopy for histone modifications to discover that $\mathrm{H} 3 \mathrm{~K} 27$ trimethylation was globally reduced in these tumors. Despite global reduction in H3K27me3, ChIP-sequencing for $\mathrm{H} 3 \mathrm{~K} 27 \mathrm{me} 3$ revealed genomic enrichment at several loci important for neurodevelopment. In a cohort of 195 childhood posterior fossa ependymoma samples, reduction in $\mathrm{H} 3 \mathrm{~K} 27 \mathrm{me} 3$ was associated with worse prognosis $(\mathrm{p}<0.0001)$. Comparison of DNA methylation and genome-wide H3K27me3 enrichment from both H3K27M mutant diffuse intrinsic pontine gliomas and childhood ependymomas patient samples showed many similarities and pointed to factors that regulate radial glial (neuronal stem cells) development. Human radial glial cells during development exhibit low $\mathrm{H} 3 \mathrm{~K} 27 \mathrm{me} 3$. Ependymomas with reduced H3K27me3 corresponded to younger children $(\mathrm{p}<0.0001)$, arose mainly from the roof of the forth ventricle, exhibited increased evidence of radiologic and histopathologic invasion 
and exhibited clinical features consistent with previously described PFA subgroup of ependymomas. Together these data suggest that the epigenetics of the developing posterior fossa are closely related to the pathogenesis of childhood brain tumors and have clinical implications for biomarker development and to inform epigenetic approaches to treat PF ependymomas.

EPND-09. THE ONCOGENIC EFFECT OF C11ORF95-RELA FUSION MOSTLY DERIVES FROM FACTOR OTHER THAN NF-KB ACTIVATION IN SUPRATENTORIAL EPENDYMOMA Tatsuya Ozawa $^{1,2}$, Sonali Arora ${ }^{2}$, Frank Szulzewsky ${ }^{2}$, and

Eric Holland ${ }^{2} ;{ }^{1}$ National Cancer Center Research Institute, Tokyo, Japan, ${ }^{2}$ Fred Hutchinson Cancer Research Center, Seattle, WA, USA.

Recent deep molecular analyses of Ependymomas (EPNs) have revealed that supratentorial ependymomas (ST-EPNs) consisted of two different molecular subgroups denoted as ST-EPN-YAP1 and -RELA which associates with YAP1- and RELA-involved fusion gene, respectively. These fusion genes may provide significant and distinct therapeutic insights for each EPN subtypes. ST-EPN-RELA subgroup associates with C11orf95RELA fusion gene (CR). The presence of RELA as one component of the CR leads to the suggestion that NF-kB activity may be essential for oncogenesis and a viable therapeutic target in these tumors. So far, we have demonstrated that the CR was a potent oncogene which was capable of inducing human EPN-like tumor in mice. However it still remains to be cleared whether NF-kB/RelA activity involved in the CR-induced tumor formation. Therefore, we undertook an extensive analysis of the connection between NF-kB/RelA activity and oncogenesis for the CR. We created various CR mutants with modification(s) in phosphorylation or acetylation site of RelA portion and tested their oncogenic potential in mice. These mutants presented superior or inferior tumor forming potential than the CR. When NF-kB/RelA activity of these mutants was measured with luciferase reporter assay in cultured fibroblasts, prominent NF-kB activity was not observed in any CR mutants and there was no correlation between NF-kB/RelA activity and tumor forming potential. Further when NF-kB activity of these CR mutants was examined using cultured fibroblasts and mouse tumors in RNA-seq, minimal NF-kB activity was observed in these mutants but not correlated with their tumor forming potential as well. These results suggested that NF-kB/RelA activity is unlikely essential for the process of tumor initiation in the CR-induced EPN formation, and the oncogenic effect of CR mostly derives from factor other than NF-kB activation. Therefore we will need further elaborate investigation whether NF-kB/RelA pathway is a right therapeutic target for these tumors.

\section{EPND-10. PRE- AND PERINATAL FACTORS ASSOCIATED WITH DEVELOPMENT OF PEDIATRIC EPENDYMOMA: RESULTS OF CASE-CONTROL ANALYSIS}

Cassie Kline $^{1}$, Libby Morimoto ${ }^{2}$, Joseph Wiemels ${ }^{1}$, Catherine Metayer ${ }^{2}$, and Kyle Walsh ${ }^{1} ;{ }^{1}$ University of California, San Francisco, San Francisco, CA, USA, ${ }^{2}$ University of California, Berkeley, Berkeley, CA, USA.

BACKGROUND: Ependymoma is the 3rd most common brain tumor in children. Few studies have investigated factors contributing to development of pediatric ependymoma. We investigate pre- and perinatal factors associated with ependymoma diagnosis in the largest, multi-ethnic cohort assembled to date. METHODS: A population-based, case-control analysis was nested within the California Cancer Registry. Cases and controls were born in California between 1978 and 2009 and linked to birth data from the California Department of Public Health vital statistics unit. Cases were diagnosed between 0-19 years of age and matched to four controls based on sex, birth year, and race/ethnicity. A total of 475 cases were included, matched to 1900 controls. RESULTS: Ependymoma cases included 273 $(57 \%)$ males. Most frequent age at diagnosis was $0-5$ years $(42 \%)$. The most common tumor histology based on ICD-O-3 code was ependymoma, NOS $(50 \%)$. When grade was reported, grade 4 was most common $(24 \%)$. Birth weight and gestational age were not different between cases and controls. Mean maternal age for cases was 28.7 years versus 27.5 years for controls $(p=0.0003)$. Each five-year increase in maternal age was associated with a 1.18 -fold increased risk of ependymoma $(\mathrm{p}<0.001)$. Mean paternal age for cases was 31.3 years versus 30.3 years in controls $(\mathrm{p}=0.01)$. Older paternal age was associated with increased incidence of grade 4 lesions $(\mathrm{p}=0.01)$ Patient age at diagnosis differed by tumor histology and grade, with younger age associated with higher tumor grade $(\mathrm{p}=0.0001)$. Notable gender differences occurred across histology, grade, and anatomic site. Incidence of pregnancy, labor, or congenital abnormalities was similar between cases and controls. CONCLUSIONS: Older parental age at birth is associated with the development of ependymoma in case-control analyses, with the strongest effect observed for maternal age. Differences in patient gender and age are seen across groupings of histology, grade, and anatomic site.
EPND-11. P16 LOSS AND E2F/CELL CYCLE DEREGULATION IN INFANT EPENDYMOMA

Andrew Donson ${ }^{1,2}$, Seth Lummus ${ }^{3}$, Rajeev Vibhakar ${ }^{1,2}$

Nicholas Foreman ${ }^{1,2}$, and BK Kleinshmidt-DeMasters ${ }^{3} ;{ }^{1}$ Dept. Pediatrics, University of Colorado Anschutz Medical Campus, Aurora, CO, USA, ${ }^{2}$ Morgan Adams Foundation Pediatric Brain Tumor Research Program, Children's Hospital Colorado, Aurora, CO, USA, ${ }^{3}$ Dept. Pathology, University of Colorado Anschutz Medical Campus, Aurora, CO, USA.

BACKGROUND: Ependymomas in infants less than 1 year of age (iEPN) have a poorer clinical outcome than EPNs in older children. While radiation therapy is the standard of care for the latter, it is withheld in iEPN to avoid neurotoxicity to immature brain. It is unknown whether the adverse outcome in iEPNs is due to treatment differences or aggressive biology. We examined this question using molecular profiling. METHODS: Six anaplastic posterior fossa (PF) iEPNs had transcriptomic analysis and fluorescence in situ hybridization for loss of $\mathrm{p} 16$ and gains of $1 \mathrm{q}$ sequences. Results were compared with anaplastic PF EPNs from older children and findings validated by immunohistochemistry. RESULTS: All 6 iEPNs grouped within EPN PF subgroup A (PFA). Further transcriptomic analyses identified E2F targets and G2M checkpoint as the most enriched gene sets in iEPN, which was validated in a larger independent cohort. Accordingly, MIB-1 immunohistochemistry demonstrated a higher mitotic rate in iEPNs than noninfant anaplastic EPN PFA. Genetic analyses demonstrated that loss of p16 was almost exclusive to iEPN, and that none harbored 1q gains. KaplanMaier analysis confirmed the poorer clinical outcome of the iEPN cohort. CONCLUSIONS: Biological differences, characterized by genetic loss of p16 without gains of $1 \mathrm{q}$ in iEPNs, as well as deregulated E2F target gene transcription, are indicative of deregulated p16-CDK4/6-pRB-E2F pathway activity. This may underlie the poor clinical outcome seen in this group of iEPNs, rather than the withholding of radiation therapy. Results suggest a potential actionable targeted therapy for iEPN, namely CDK4/6 inhibitors such as palbociclib, which has shown favorable safety profiles in children.

\section{EPND-12. TYROSINE KINASE INHIBITORS AXITINIB, IMATINIB} AND PAZOPANIB ARE SELECTIVELY POTENT IN EPENDYMOMA Andrew Donson ${ }^{1,2}$, Elliot Werner ${ }^{1,2}$, Vladimir Amani ${ }^{1,2}$

Andrea Griesinger ${ }^{1,2}$, Davis Witt ${ }^{1,2}$, Anandani Nellan ${ }^{1,2}$, Rajeev Vibhakar ${ }^{1,2}$, Todd Hankinson ${ }^{3}$, Michael Handler ${ }^{3}$, Kathleen Dorris ${ }^{1,2}$, and

Nicholas Foreman ${ }^{1,2} ;{ }^{1}$ Dept. Pediatrics, University of Colorado Anschutz Medical Campus, Aurora, CO, USA, ${ }^{2}$ Morgan Adams Foundation Pediatric Brain Tumor Research Program, Children's Hospital Colorado, Aurora, CO, USA, ${ }^{3}$ Dept. Neurosurgery, University of Colorado Anschutz Medical Campus, Aurora, CO, USA.

Reproducibly effective chemotherapy has yet to be identified for ependymoma (EPN), partly due to a lack of models for preclinical drug screening. Treatment of EPN has therefore changed little over the last 40 years and is essentially limited to surgery and radiation. Recurrence risk is high, particularly in those EPN harboring chromosome 1q gain. To address this, we have established 2 posterior fossa $1 \mathrm{q}+$ EPN cell lines and we have utilized these in a high-throughput drug screen of FDA-approved oncology drugs. Treatment effect was measured using a tritiated-thymidine incorporation proliferation assay and EPN-selective sensitivity was determined by comparison to a pane of other pediatric brain tumor types (medulloblastoma, glioblastoma, DIPG and AT/RT). Using this approach, we identified a group of 3 small molecule tyrosine kinase inhibitors (TKIs) that showed greater potency in EPN than control cell lines. These TKIs - namely axitinib, imatinib and pazopanib - commonly target VEGFR, PDGFR and c-kit. Of note, axitinib has been proposed for an upcoming phase 1 trial for recurrent pediatric brain tumors including EPN. Studies of axitinib in other tumors demonstrated a beneficial immunomodulatory effect, of particular relevance in EPN given the key role of immunity in this tumor. Axitinib may therefore have a dual mechanism of tumor control in EPN. We selected this drug for further evaluation in our preclinical model of EPN. Dose curve analysis confirmed that axitinib more is potent in EPN than other cell types. Transcriptomic analysis of axitinib treated EPN revealed a decrease in mitosis-related gene expression and in increase in type-I interferon-associated gene expression. Further screening studies identified treatments that showed synergistic effects in EPN, most notably cisplatin. Collectively these data support clinical evaluation of axitinib for treatment EPN and provide information of potentially synergistic treatment combinations that might overcome single agent resistance.

EPND-13. YAP1-MAMLD1 FUSIONS ALONE ARE SUFFICIENT TO FORM SUPRATENTORIAL EPENDYMOMA-LIKE TUMORS IN MICE Kristian W. Paitler ${ }^{1,2}$, Sebastian Brabetz ${ }^{1}$, David Capper ${ }^{3,4}$, Mikaella Vouri $^{1}$, Monika Mauermann ${ }^{1}$, Norman Mack ${ }^{1}$, Laura Sieber ${ }^{1}$, Hendrik Witt ${ }^{1,2}$ David T. W. Jones ${ }^{1}$, Andrey Korshunov ${ }^{3,4}$, Stefan M. Pfister ${ }^{1,2}$, Marcel Kool ${ }^{1}$, and Daisuke Kawauchi ${ }^{1}$; ${ }^{1}$ Division of Pediatric Neurooncology, German Cancer Research Center, Heidelberg, Germany,

${ }^{2}$ Department of Pediatric Oncology, Hematology and Immunology, 